\title{
EULER CHARACTERISTIC OF IMAGINARIES IN O-MINIMAL STRUCTURES
}

\author{
SOFYA KAMENKOVICH AND YA'ACOV PETERZIL
}

\begin{abstract}
We define the notion of Euler characteristic for definable quotients in an arbitrary o-minimal structure and prove some fundamental properties.
\end{abstract}

\section{INTRODUCTION}

Let $\mathcal{M}$ be an arbitrary o-minimal structure expanding a dense linear order without end points. Given a definable set $X$ in $\mathcal{M}$ and a definable equivalence relation $E$ on $X$, we call $\frac{X}{E}$ a definable quotient. As was pointed out in [6], o-minimal structures in general do not admit elimination of imaginaries. However, in [2] the following was asked:

Question Is it true that for every definable quotient $\frac{X}{E}$ there exist a definable set $D$, possibly over additional parameters, and a definable surjection $f: X \rightarrow D$ such that for every $x_{1}, x_{2} \in X, x_{1} E x_{2}$ iff $f\left(x_{1}\right)=f\left(x_{2}\right)$ ? We call such a $D$ a coding set for $\frac{X}{E}$.

As we know, if $\mathcal{M}$ expands an ordered group and at least one nonzero element is named then every definable quotient has a coding set defined over the same set of parameters. It was also shown in [2] that if $\frac{X}{E}$ admits a definable group structure then it has a coding set (possibly, over additional parameters). Recently, Johnson found an ingenious example which shows that not every definable quotient has a coding set (see [3]), so o-minimal structures in general do not eliminate imaginaries, even in this weaker sense.

Thus, there is a need to develop a better understanding of $\mathcal{M}^{e q}$ which goes beyond the study of definable sets.

Two important integral invariants in o-minimal structures, associated to definable sets are dimension and Euler characteristic (see for example [7] for its beautiful applications for definable groups). The definition of dimension for definable quotients was carried out in [5] and

Date: September 3, 2014.

Key words and phrases. O-minimality, Euler Characteristic of Imaginaries, definable quotients.

This article was part of the M.Sc thesis of the first author at the University of Haifa. We thank Assaf Hasson and Victor Harnik for their suggestions. 
[4]. The goal of this note is to treat Euler characteristic in a similar fashion.

Recall, [1], that in o-minimal structures, the definition of the Euler characteristic, $\chi(U)$, for a definable set $U$ is based on the cell decomposition theorem, and is defined by

$$
\chi(U)=\sum_{C \in \mathcal{C}}(-1)^{\operatorname{dim}(C)} .
$$

where $\mathcal{C}$ is any partition of $U$ into cells.

Notation Given a definable quotient $\frac{U}{E}$, and $u \in U$, we write $[u]_{E}$ (or sometimes just $[u]$ ) for its $E$-equivalence class. We will denote by $\chi^{e q}\left(\frac{U}{E}\right)$ its Euler characteristic, whose definition we describe below.

The main idea. Our initial expectation was that the extension of the definition of Euler characteristic from definable sets to definable quotients will be purely combinatorial, based on the following expected equality, in the simplest case, where all classes in $U$ have the same Euler characteristic $k$.

$$
\chi(U)=\chi^{e q}\left(\frac{U}{E}\right) \cdot k
$$

However, this equality (which indeed, we prove in 3.1) does not help us to determine $\chi^{e q}\left(\frac{U}{E}\right)$ in the case $k=0$. We will bypass this difficulty by applying first a uniform version of the cell decomposition theorem and replacing the original quotient $\frac{U}{E}$ by another one, in which every class is a cell, so has nonzero Euler characteristic.

Our goal then in this note is to define $\chi^{e q}$ and prove its fundamental properties. First some definitions:

Definition 1.1. For a definable quotient $\frac{U}{E}$, a subset $Y \subseteq \frac{U}{E}$ is called definable if its pullback

$$
\bar{Y}=\left\{x \in U:[x]_{E} \in X\right\}
$$

definable set.

For $\frac{X}{E}$ and $\frac{Y}{F}$ definable quotients, a function $f: \frac{X}{E} \rightarrow \frac{Y}{F}$ is called definable if its pull-back to $X \times Y$,

$$
\bar{f}=\{(x, y) \in X \times Y: f([x])=[y]\}
$$

is a definable set.

For $\frac{U}{E}$ a definable quotient and $Y \subseteq \frac{U}{E}$ a definable set, we will write $\chi^{e q}(Y)$ to mean $\chi^{e q}\left(\frac{\bar{Y}}{E^{\prime}}\right)$ where $E^{\prime}$ is the restriction of $E$ to the set $\bar{Y}$.

We will prove:

1. If every E-class is a singleton then $\chi^{e q}\left(\frac{U}{E}\right)=\chi(U)$. Namely, our definition of $\chi^{e q}$ extends that of $\chi$.

2. If $\frac{U}{E}$ is a definable quotient and $\left|\frac{U}{E}\right|=1$ then $\chi^{e q}\left(\frac{U}{E}\right)=1$. 
3. If $\frac{U}{E}$ is a definable quotient, $Y_{1}, Y_{2} \subseteq \frac{U}{E}$ definable sets, then

$$
\chi^{e q}\left(Y_{1} \cup Y_{2}\right)=\chi^{e q}\left(Y_{1}\right)+\chi^{e q}\left(Y_{2}\right)-\chi^{e q}\left(Y_{1} \cap Y_{2}\right)
$$

4. Let $\frac{U}{E}$ and $\frac{V}{F}$ be definable quotients and let $f: \frac{U}{E} \rightarrow \frac{V}{F}$ be definable surjective function, such that for every $[v] \in \frac{V}{F}$ we have $\chi^{e q}\left(f^{-1}\left([\bar{v}]_{F}\right)\right)=e$, then $\chi^{e q}\left(\frac{U}{E}\right)=\chi^{e q}\left(\frac{V}{F}\right) \cdot e$. In particular, $\chi^{e q}$ is invariant under definable bijections.

We also prove additional properties of $\chi^{e q}$ such as definability in parameters..

\section{Euler Characteristic of Imaginaries}

2.1. Preliminaries. We fix an o-minimal expansion $\mathcal{M}=\langle M,<, \cdots\rangle$ of a dense linear order without endpoints. All definability below is taken in $\mathcal{M}$.

The first clause of the claim below is just [1, Prop. 4.2.10].

Claim 2.1. Let $\left\{X_{t}: t \in T\right\}$ be definable family of subsets of $M^{n}$, $e \in \mathbb{Z}$, then set $T_{e}:=\left\{t \in T: \chi\left(X_{t}\right)=e\right\}$ is definable. Moreover, there exists a bound $k$ such that for every $t \in T,\left|\chi\left(X_{t}\right)\right| \leq k$

Let us prove the last clause: Consider a cell decomposition $\mathcal{C}$ of the set

$$
X=\left\{(t, x) \in T \times M^{n}: x \in X_{t}\right\} .
$$

Note that if $C \subseteq T \times M^{n}$ is a cell, then for each $t \in T$, the set $C_{t}=\left\{x \in M^{n}:(t, x) \in C\right\}$ is either a cell or empty. Thus, for every $t \in T$ the decomposition $\mathcal{C}$ induces a decomposition of $X_{t}$ into (possibly empty) cells $\left\{C_{t}: C \in \mathcal{C}\right\}$, with at most as many cells as in $\mathcal{C}$. By definition, it follows that $\left|\chi\left(X_{t}\right)\right| \leq|\mathcal{C}|$.

Corollary 2.2. Let $f: U \rightarrow M^{k}$ be a definable function for a definable set $U \subseteq M^{n}$. If $\chi\left(f^{-1}(a)\right)=e$ for each $a \in f(U)$, then

$$
\chi(U)=e \cdot \chi(f(U)) .
$$

Proof. Define $\pi_{1}: \operatorname{graph}(f) \rightarrow f(U)$. For every $a \in f(U), \pi^{-1}(a)=$ $f^{-1}(a) \times\{a\}$. We have

$$
\chi\left(\pi^{-1}(a)\right)=\chi\left(f^{-1}(a) \times\{a\}\right)=\chi\left(f^{-1}(a)\right)=e .
$$

So by $[1,4.2 .11] \chi(\operatorname{graph}(f))=e \cdot \chi(f(U))$. Since $U$ and $\operatorname{graph}(f)$ are in definable bijection we get $\chi(U)=e \cdot \chi(f(U))$.

Now if we define $\pi_{2}: \operatorname{graph}(f) \rightarrow U, \pi_{2}$ is a bijection hence, by $[1$, Th. 4.2.4] we get that $\chi(U)=\chi(\operatorname{graph}(f))=e \cdot \chi(f(U))$.

Definition 2.3. For a definable quotient $\frac{U}{E}$ we define

$$
U_{(k)}^{E}=\left\{u \in U \mid \chi\left([u]_{E}\right)=k\right\}
$$

and omit the superscript $E$ when it is clear from the context. 
We will use a uniform version of cell decomposition theorem (see [2, Lemma 9.1]).

Theorem 2.4. Let $\left\{X_{t}: t \in T\right\}$ be a definable family of subsets of $M^{n}$. Then there are $k \in \mathbb{N}$ and definable families of sets in $M^{n}$

$$
\left\{X_{1, t}: t \in T\right\}, \ldots,\left\{X_{k, t}: t \in T\right\}
$$

such that:

(i) Each $X_{i t}$ is either a cell or the empty set.

(ii) For every $a \in T, X_{a}=X_{1, a} \sqcup \ldots \sqcup X_{k, a}$, and

(iii) For every $a, b \in T$, if $X_{a}=X_{b}$ then $X_{i, a}=X_{i, b}$ for $i=1, \ldots, k$.

By applying the above proposition to the family of equivalence classes in a definable quotient we obtain:

Corollary 2.5. Let $E$ be a definable equivalence on a definable set $U \subseteq M^{n}$. Then there are $k \in \mathbb{N}$ and definable families of sets

$$
\left\{C_{1, u}: u \in U\right\}, \ldots,\left\{C_{k, u}: u \in U\right\}
$$

such that:

(i) Each $C_{i, u}$ is either a cell or the empty set

(ii) For every $u \in U,[u]_{E}=C_{1, u} \sqcup \ldots \sqcup C_{k, u}$, and

(iii)For every $u, v \in U$, if $[u]_{E}=[v]_{E}$ then $C_{1 u}=C_{1, v}, \ldots, C_{k, u}=C_{k, v}$.

2.2. The definition of Euler characteristic for imaginaries. In order to motivate our definition, assume first that the quotient $\frac{E}{U}$ has a coding set $\mathrm{D}$. Namely we have a definable surjection $f: U \rightarrow D$ s.t. $f\left(u_{1}\right)=f\left(u_{2}\right)$ iff $u_{1} E u_{2}$. Our definition should imply $\chi^{e q}\left(\frac{U}{E}\right)=$ $\chi(D)$. Assume in addition that every $E$-class has Euler characteristic $k$. Then, by Theorem 2.2, we have $k \cdot \chi(D)=\chi(U)$ and hence we expect $\chi^{e q}\left(\frac{U}{E}\right)=\chi(D)=\chi(U) / k$. We would have liked to use this simple calculation as a basis for our definition of $\chi^{e q}$ and define

$$
\chi^{e q}\left(\frac{U}{E}\right)=\sum_{k} \frac{\chi\left(U_{(k)}\right)}{k}
$$

but a problem arises when $k=0$, namely if $U_{(0)} \neq \emptyset$.

To bypass this problem we will use the uniform cell decomposition theorem and replace each definable quotient by one in which all classes are cells and hence of nonzero Euler characteristic.

Theorem 2.6. Let $\frac{U}{E}$ be a definable $M$-quotient. Then there is a definable quotient $\frac{V}{F}$ and a definable bijection $f: \frac{U}{E} \rightarrow \frac{V}{F}$ such that every equivalence class of $F$ is a cell in $V$.

Proof. By 2.5 there are k definable families

$$
\left\{C_{1, u}: u \in U\right\}, \ldots,\left\{C_{k, u}: u \in U\right\}
$$

such that:

(i) Each $C_{i u}, i=1, \ldots, k$ is either a cell or the empty set 
(ii) For every $u \in U,[u]=C_{1 u} \sqcup \ldots \sqcup C_{k u}$ and

(iii) For every $u, v \in U$, if $[u]_{E}=[v]_{E}$ then $C_{i, u}=C_{i, v}$, for $i=1, \ldots, k$.

We now choose, for each $u \in U$, the first $i=1, \ldots, k$ for which $C_{i, u}$ is nonempty, and let $V$ be the union of all these $C_{i, u}$ 's, and $F$ be the restriction of $E$ to $V$. Notice that the choice of $C_{i, u}$ depends only on $[u]$, so each class $[u]$ will contain a single cell. The map which sends a class $[u]$ to the first $C_{i, u}$ which is nonempty is a definable bijection between $\frac{U}{E}$ and $\frac{V}{F}$.

We will also need a uniform version of the above.

Definition 2.7. Assume that $\left\{U_{t}: t \in T\right\},\left\{E_{t}: t \in T\right\}$ are definable families, such that $E_{t}$ is an equivalence relation on $U_{t}$ for all $t \in T$. Then we say that $\left\{\frac{U_{t}}{E_{t}}: t \in T\right\}$ is definable family of quotients.

Similarly to the proof of Theorem 2.6 one obtains:

Theorem 2.8. Let $\left\{\frac{U_{t}}{E_{t}}: t \in T\right\}$ be a definable family of $M$-quotients. Then there exists a definable family of quotients $\left\{\frac{V_{t}}{F_{t}}: t \in T\right\}$ and a definable family of functions $\left\{f_{t}: t \in T\right\}$ such that

(1) For each $t \in T$, every $F_{t}$-class is a cell.

(2) For each $t \in T, f_{t}: \frac{U_{t}}{E_{t}} \rightarrow \frac{V_{t}}{F_{t}}$ is a definable bijection.

We are now ready to formulate the definition of $\chi^{e q}\left(\frac{U}{E}\right)$.

Definition 2.9. Euler characteristic for definable quotients.

(1) Let $\frac{V}{F}$ be a definable M-quotient such that every F-class is a cell, then define:

$$
\chi^{e q}\left(\frac{V}{F}\right):=\chi\left(V_{(1)}\right)-\chi\left(V_{(-1)}\right)
$$

(2) Let $\frac{U}{E}$ be a definable quotient. Take some definable bijection $f: \frac{U}{E} \rightarrow \frac{V}{F}$ such that $[v]_{F}$ is cell for every $v \in V$ and define:

$$
\chi^{e q}\left(\frac{U}{E}\right):=\chi^{e q}\left(\frac{V}{F}\right)
$$

Note, the above definition agrees with our initial intuition since $\chi\left(U_{(1)}\right)-\chi\left(U_{(-1)}\right)=\frac{\chi\left(U_{(1)}\right)}{1}+\frac{\chi\left(U_{(-1)}\right)}{-1}=\sum_{k=1,-1} \frac{\chi\left(U_{(k)}\right)}{k}$.

It is left to see that Clause (2) gives a well defined notion of $\chi^{e q}\left(\frac{U}{E}\right)$, independent of the choice of $V$ and $F$. This is the content of the next section.

2.3. $\chi^{e q}$ is well defined. We first prove a result about the Euler characteristic of definable sets.

Proposition 2.10. Assume $\frac{V}{F}, \frac{W}{H}$ are definable $M$-quotients, such that for every $v \in V \chi\left([v]_{F}\right)=m$, for every $w \in W, \chi\left([w]_{H}\right)=n$ and $G: \frac{V}{F} \rightarrow \frac{W}{H}$ is a definable bijection. Then $\chi(V) \cdot n=\chi(W) \cdot m$ 
Proof. Let

$$
\bar{G}:=\left\{(v, w) \subseteq V \times W \mid G\left([v]_{F}\right)=[w]_{H}\right\}
$$

If we define $\pi_{1}: \bar{G} \rightarrow V, \pi_{1}(v, w)=v$, then

$$
\pi_{1}^{-1}\left(v_{0}\right)=\left\{\left(v_{0}, w\right) \mid G\left(\left[v_{0}\right]_{F}\right)=[w]_{H}\right\} \subseteq\left\{v_{0}\right\} \times W .
$$

Then, we have $\left(v_{0}, w\right) \in \pi_{1}^{-1}\left(v_{0}\right)$ iff $[w]_{H} \in G\left(\left[v_{0}\right]_{F}\right)$, hence $\pi_{1}^{-1}\left(v_{0}\right)=\left\{v_{0}\right\} \times G\left(\left[v_{0}\right]_{F}\right)$. By assumption we get

$$
\chi\left(\pi_{1}^{-1}\left(v_{0}\right)\right)=\chi\left(G\left(\left[v_{0}\right]_{F}\right)\right)=n .
$$

This equality is true for all $v_{0} \in V$, so by 2.2 , applied to $\pi_{1}$,

$$
\chi(\bar{G})=n \cdot \chi(V) \text {. }
$$

Now if we look at $G^{-1}$, then by symmetry we obtain

$$
\chi(\bar{G})=m \cdot \chi(W) .
$$

By (1) and (2) we get

$$
n \cdot \chi(V)=\chi(\bar{G})=m \cdot \chi(W) .
$$

The following clearly implies that our notion of $\chi^{e q}$ is well defined.

Theorem 2.11. Assume that $V \subseteq M^{n}$ and $W \subseteq M^{r}$ are definable sets. Let $\frac{V}{F}$ and $\frac{W}{H}$ be definable quotients, such that every $E$-equivalence class and every $F$-equivalence class is a cell. Assume that $G: \frac{V}{F} \rightarrow \frac{W}{H}$ is a definable bijection, then

$$
\chi\left(V_{(1)}\right)-\chi\left(V_{(-1)}\right)=\chi\left(W_{(1)}\right)-\chi\left(W_{(-1)}\right)
$$

Proof. Since every class is a cell, then by definition of Euler characteristic for sets we have: $\chi\left([v]_{F}\right)= \pm 1$ and $\chi\left([w]_{H}\right)= \pm 1$. Then $V=V_{(1)} \bigsqcup V_{(-1)}$ and $W=W_{(1)} \bigsqcup W_{(-1)}$. For $k, l \in\{ \pm 1\}$ define:

$$
V_{k}^{l}:=\left\{v \in V \mid \chi\left([v]_{F}\right)=k \wedge \chi\left(G\left([v]_{F}\right)\right)=l\right\} .
$$

Since $G$ is a bijection, for every $w \in W, G^{-1}\left([w]_{H}\right)$ is a single $F$-class so we can define:

$$
W_{k}^{l}:=\left\{w \in W \mid \chi\left(G^{-1}\left([w]_{H}\right)\right)=k \wedge \chi\left([w]_{H}\right)=l\right\} .
$$

Notice that the restriction of $G$ to $\frac{V_{k}^{l}}{F}$ induces a bijection with $\frac{W_{k}^{l}}{H}$. Let $G_{k, l}$ denote this restriction.

By Proposition 2.10

$$
G_{k, l}: \frac{V_{k}^{l}}{F} \rightarrow \frac{W_{k}^{l}}{H}
$$

$$
l \cdot \chi\left(V_{k}^{l}\right)=k \cdot \chi\left(W_{k}^{l}\right),
$$

so $\chi\left(V_{k}^{l}\right)=\chi\left(W_{k}^{l}\right)$ if and only if $k=l$ (and otherwise they are opposite). Now, $V_{(k)}=V_{k}^{1} \sqcup V_{k}^{-1}$ and $W_{(k)}=W_{1}^{k} \sqcup W_{-1}^{k}$, so we obtain.

$$
\chi\left(V_{(1)}\right)-\chi\left(V_{(-1)}\right)=\chi\left(V_{1}^{1}\right)+\chi\left(V_{1}^{-1}\right)-\left(\chi\left(V_{-1}^{1}\right)+\chi\left(V_{-1}^{-1}\right)\right)=
$$




$$
\chi\left(W_{1}^{1}\right)-\chi\left(W_{1}^{-1}\right)+\chi\left(W_{-1}^{1}\right)-\chi\left(W_{-1}^{-1}\right)=\chi\left(W_{(1)}\right)-\chi\left(W_{(-1)}\right) .
$$

This ends the proof that $\chi^{e q}$ is well defined.

We may now conclude:

Proposition 2.12. If $f: \frac{U_{1}}{E_{1}} \rightarrow \frac{U_{2}}{E_{2}}$ is a definable bijection then

$$
\chi^{e q}\left(\frac{U_{1}}{E_{1}}\right)=\chi^{e q}\left(\frac{U_{2}}{E_{2}}\right)
$$

Proof. By definition of $\chi^{e q}$ we associate to each $\frac{U_{i}}{E_{i}}$ quotients whose classes are cells, and apply Theorem 2.11.

We end this section with the following simple observation:

Claim 2.13. Let $\frac{U}{E}$ be a definable quotient such that every class is a cell. Then:

$$
\chi^{e q}\left(\frac{U}{E}\right)=\sum_{k= \pm 1} k \cdot \chi\left(U_{(k)}\right) .
$$

\subsection{Uniform definability of $\chi^{e q}$.}

Proposition 2.14. Let $\left\{\frac{U_{t}}{E_{t}}: t \in T\right\}$ be a definable family of quotients. Then, for every integer e the set $\left\{t \in T: \chi^{e q}\left(\frac{U_{t}}{E_{t}}\right)=e\right\}$ is definable.

In particular, if $\left\{X_{t} \subseteq \frac{U}{E}, t \in T\right\}$ is definable family of subsets of $\frac{U}{E}$, then for every integer number $e$ the set $\left\{t \in T: \chi^{e q}\left(X_{t}\right)=e\right\}$ is definable.

Proof. Using Theorem 2.8, we may assume that every equivalence class in $U_{t}$ is a cell.

Claim 2.15. Let $\left\{\frac{U_{t}}{E_{t}}: t \in T\right\}$ be family of definable quotients where every equivalence class is a cell, then there is $p \in \mathbb{Z}$ such that for every $t \in T,\left|\chi^{e q}\left(\frac{U_{t}}{E_{t}}\right)\right| \leq p$

Proof. By definition, the families $\left\{U_{t}: t \in T\right\}$ and $\left\{E_{t}: t \in T\right\}$ are definable, hence by Claim 2.1, $\left\{U_{t(1)} \mid t \in T\right\},\left\{U_{t(-1)} \mid t \in T\right\}$ are definable families of sets. Hence by 2.1 there exist bound $k_{1}$ for $\left|\chi\left(U_{t(1)}\right)\right|$ and bound $k_{2}$ for $\left|\chi\left(U_{t(-1)}\right)\right|$, hence $k_{1}+k_{2}$ is a bound for $\left|\chi^{e q}\left(\frac{U_{t}}{E_{t}}\right)\right|$.

We now return to the proposition:

By using the construction from previous claim let us build exactly the formula that define the set $\left\{t \in T: \chi^{e q}\left(\frac{U_{t}}{E_{t}}\right)=e\right\}$. Let us define

then

$$
\begin{gathered}
\varphi_{e}^{+}(t):=\left\{t \in T: \chi\left(U_{t(1)}\right)=e\right\}, \\
\varphi_{r}^{-}(t):=\left\{t \in T: \chi\left(U_{t(-1)}\right)=r\right\},
\end{gathered}
$$

$$
\mu_{e}(t):=\bigvee_{-p \leq k, k-e \leq p} \varphi_{k}^{+}(t) \wedge \varphi_{k-e}^{-}(t)
$$

is the desired formula.

This ends the proof of Proposition 2.14. 
2.5. Properties of $\chi^{e q}$. Note that if $E$ is the equality relation on $U$ then every class is a singleton, so $U=U_{(1)}$ and hence $\chi^{e q}\left(\frac{U}{E}\right)=\chi(U)$. This shows that our definition of $\chi^{e q}$ is indeed an extension of $\chi$. More generally we can now conclude:

Lemma 2.16. If $\frac{U}{E}$ is a definable $M$-quotient and $Y$ is coding set of $\frac{U}{E}$, then $\chi^{e q}\left(\frac{U}{E}\right)=\chi(Y)$.

Proof. Since have a definable bijection between $\frac{U}{E}$ and $\frac{Y}{=}$, we can apply Proposition 2.12 together with the above observation and conclude $\chi^{e q}\left(\frac{U}{E}\right)=\chi(Y)$.

In particular, we have:

Claim 2.17. Let $\frac{U}{E}$ be a definable $M$-quotient. If $\frac{U}{E}$ is a singleton then $\chi^{e q}\left(\frac{U}{E}\right)=1$.

Theorem 2.18. Assume $\frac{U}{E}$ definable quotient. $Y_{1}, Y_{2} \subseteq \frac{U}{E}$ definable sets. Then

$$
\chi^{e q}\left(Y_{1} \cup Y_{2}\right)=\chi^{e q}\left(Y_{1}\right)+\chi^{e q}\left(Y_{2}\right)-\chi^{e q}\left(Y_{1} \cap Y_{2}\right) .
$$

Proof. It is sufficient to prove the result when $Y_{1} \cap Y_{2}=\emptyset$. By 2.6, we may assume that every class is a cell.

Since $E$ is equivalence relation and $Y_{1}, Y_{2} \subseteq \frac{U}{E}$, are disjoint sets, the corresponding subsets of $U, \overline{Y_{1}}$ and $\overline{Y_{2}}$ are also disjoint. It easily follows, for $k= \pm 1$, that

$$
{\overline{Y_{1}}}_{(k)} \cup{\overline{Y_{2}}}_{(k)}=\left(\overline{Y_{1}} \cup \overline{Y_{2}}\right)_{(k)} .
$$

By definition of $\chi^{e q}$ (and Claim 2.13) we can write:

$$
\begin{gathered}
\chi^{e q}\left(Y_{1}\right)+\chi^{e q}\left(Y_{2}\right)=\sum_{k=1,-1} k \chi\left(\overline{Y_{1(k)}}\right)+\sum_{k=1,-1} k \chi\left(\overline{Y_{2}}(k)\right)= \\
=\sum_{k=1,-1} k\left(\chi\left(\bar{Y}_{1(k)}\right)+\chi\left(\overline{Y_{2}(k)}\right)\right)=\sum_{k=1,-1} k\left(\chi\left(\overline{Y_{1}} \cup \overline{Y_{2}}\right)_{(k)}\right)=\chi^{e q}\left(Y_{1} \cup Y_{2}\right) .
\end{gathered}
$$

The following is an analogue of Theorem 2.2 for Euler characteristic in quotients.

Theorem 2.19. Assume $\frac{U}{E}, \frac{V}{F}$ are definable quotients. Assume $f$ : $\frac{U}{E} \rightarrow \frac{V}{F}$ is a definable surjective function, such that for every $[v]_{F} \in$ $f\left(\frac{U}{E}\right), \chi^{e q}\left(f^{-1}\left([v]_{F}\right)\right)=e$, then

$$
\chi^{e q}\left(\frac{U}{E}\right)=\chi^{e q}\left(\frac{V}{E}\right) \cdot e .
$$

Proof. Because definable bijections between quotients preserve $\chi^{e q}$ we may assume from now on that each $E$-class in $U$ and $F$-class in $V$ is a cell. 
Remark: Assume every E-class is a cell of Euler characteristic $k \in$ $\{ \pm 1\}$ (same $k$ for all classes). Then, by definition

$$
\chi^{e q}\left(\frac{U}{E}\right)=k \cdot \chi\left(U_{(k)}\right)=k \cdot \chi(U) .
$$

We first prove the following special case.

Claim 2.20. For $U, V$ and $f$ as in the theorem, assume in addition that every E-class is a cell of Euler characteristic $k$ (so $\left.U=U_{(k)}\right)$ and every F-class is a cell of Euler characteristic $l\left(\right.$ so $\left.V=V_{(l)}\right)$.

Then

$$
\chi^{e q}\left(\frac{U}{E}\right)=\chi^{e q}\left(\frac{V}{F}\right) \cdot e .
$$

Proof. By definition $\chi^{e q}\left(f^{-1}\left([v]_{F}\right)\right)=\chi^{e q}\left(\frac{\overline{f^{-1}\left([v]_{F}\right)}}{E^{\prime}}\right)$ where $E^{\prime}$ is the restriction of $E$ to $\overline{f^{-1}\left([v]_{F}\right)}$. By the remark above,

$$
\chi^{e q}\left(f^{-1}\left([v]_{F}\right)\right)=k \cdot \chi\left(\overline{f^{-1}\left([v]_{F}\right)}\right),
$$

hence for every $v \in V, \chi\left(\overline{f^{-1}\left([v]_{F}\right)}\right)=k \cdot e$.

Consider $\bar{f} \subseteq U \times V$. For every $u \in U$, we have

$$
\{v \in V:(u, v) \in \bar{f}\}=\overline{f\left([u]_{E}\right)}
$$

(the set of elements in the class $f\left([u]_{E}\right)$ ). Also, for every $v \in V$ we have

$$
\{u \in U:(u, v) \in \bar{f}\}=\overline{f^{-1}\left([v]_{F}\right)} .
$$

By applying 2.2 to both projection maps (from $\bar{f}$ onto $U$ and $V$ ) we have

$$
\chi(\bar{f})=\chi(U) \cdot \chi\left(\overline{f\left([u]_{E}\right)}\right)=\chi(U) \cdot l
$$

and

$$
\chi(\bar{f})=\chi(V) \cdot \chi\left(\overline{f^{-1}[v]_{F}}\right)=\chi(V) \cdot k \cdot e .
$$

We therefore have: $\chi(U) \cdot l=\chi(V) \cdot k \cdot e$, hence $(k, l= \pm-1)$

$$
k \cdot \chi(U)=l \cdot \chi(V) \cdot e .
$$

Applying the Remark above once more we conclude

$$
\chi^{e q}\left(\frac{U}{E}\right)=\chi^{e q}\left(\frac{V}{F}\right) \cdot e .
$$

This ends the proof the Claim 2.20.

As a corollary one gets another special case of the theorem:

Claim 2.21. For $U, V$ and $f$ as in the theorem, assume in addition that $U=U_{(k)}$, for a fixed $k \in\{ \pm 1\}$. Then

$$
\chi^{e q}\left(\frac{U}{E}\right)=\chi^{e q}\left(\frac{V}{F}\right) \cdot e .
$$


Proof. Denote $U^{\prime}=f^{-1}\left(\frac{V_{(1)}}{F}\right), U^{\prime \prime}=f^{-1}\left(\frac{V_{(-1)}}{F}\right)$. By 2.20 and 2.18 we get

$$
\begin{gathered}
\chi^{e q}\left(\frac{U}{E}\right)=\chi^{e q}\left(U^{\prime}\right)+\chi^{e q}\left(U^{\prime \prime}\right)= \\
=e \cdot \chi^{e q}\left(\frac{V_{(1)}}{F}\right)+e \cdot \chi^{e q}\left(\frac{V_{(-1)}}{F}\right)=e \cdot\left(\chi^{e q}\left(\frac{V_{(1)}}{F}\right)+\chi^{e q}\left(\frac{V_{(-1)}}{F}\right)\right)=e \cdot\left(\chi^{e q}\left(\frac{V}{F}\right)\right) .
\end{gathered}
$$

Proof of Theorem 2.19: Consider first $f^{-1}\left([v]_{F}\right)$ for some $v \in V$ and let

$$
p=\chi^{e q}\left(f^{-1}\left([v]_{F}\right) \cap \frac{U_{(1)}}{E}\right) .
$$

Because $\chi^{e q}\left(f^{-1}\left([v]_{F}\right)\right)=e$ and $\frac{U}{E}=\frac{U_{(1)}}{E} \sqcup \frac{U_{(-1)}}{E}$, we get

$$
\chi^{e q}\left(f^{-1}\left([v]_{F}\right) \cap \frac{U_{(-1)}}{E}\right)=e-p .
$$

By 2.15, there exist $T \in \mathbb{Z}$ s.t. $\left|\chi^{e q}\left(f^{-1}\left([v]_{F}\right) \cap \frac{U_{(1)}}{E}\right)\right| \leq T$, for all $v \in V$.

For all $-T \leq p \leq T$, let

$$
\begin{gathered}
V^{p}:=\left\{v \in V \mid \chi^{e q}\left(f^{-1}\left([v]_{F}\right) \cap \frac{U_{(1)}}{E}\right)=p\right\} \\
U_{(1)}^{p}:=\left\{u \in U_{(1)} \mid f\left([u]_{E}\right) \in V^{p}\right\}
\end{gathered}
$$

Let $f_{1}^{p}: \frac{U_{(1)}^{p}}{E} \rightarrow \frac{V^{p}}{F}$ be the restriction of $\mathrm{f}$ to $\frac{U_{(1)}^{p}}{E}$. By 2.21 we get

$$
\chi^{e q}\left(\frac{U_{(1)}^{p}}{E}\right)=p \cdot \chi^{e q}\left(\frac{V^{p}}{F}\right) .
$$

Since $T$ is an upper bound on $\left|\chi^{e q}\left(f^{-1}\left([v]_{F}\right) \cap \frac{U_{(1)}}{E}\right)\right|$ we get

$$
\begin{gathered}
\frac{U_{(1)}}{E}=\bigsqcup_{-T \leq p \leq T}\left(\frac{U_{(1)}^{p}}{E}\right) . \\
\chi^{e q}\left(\frac{U_{(1)}}{E}\right)=\sum_{p=-T}^{T} \chi^{e q}\left(\frac{U_{(1)}^{p}}{E}\right)=\sum_{p=-T}^{T} p \cdot \chi^{e q}\left(\frac{V^{p}}{F}\right)
\end{gathered}
$$

For every $p \in T$ and $v \in V$ we have

$$
\chi^{e q}\left(f^{-1}\left([v]_{F}\right) \cap \frac{U_{(-1)}}{E}\right)=e-p \Leftrightarrow \chi^{e q}\left(f^{-1}\left([v]_{F}\right) \cap \frac{U_{(1)}}{E}\right)=e .
$$

Thus, the set of all $v \in V$ such that $\chi^{e q}\left(f^{-1}\left([v]_{F}\right) \cap \frac{U_{(-1)}}{E}\right)=e-p$ is exactly $V^{p}$. Again, by Claim 2.21,

$$
\chi^{e q}\left(\frac{U_{(-1)}^{p}}{E}\right)=(e-p) \cdot \chi^{e q}\left(\frac{V^{p}}{F}\right) .
$$


Finally,

$$
\begin{gathered}
\chi^{e q}\left(\frac{U}{E}\right)=\chi^{e q}\left(\frac{U_{(1)}}{E}\right)+\chi^{e q}\left(\frac{U_{(-1)}}{E}\right)=\sum_{p=-T}^{T} \chi^{e q}\left(\frac{U_{(1)}^{p}}{E}\right)+\sum_{p=-T}^{T} \chi^{e q}\left(\frac{U_{(-1)}^{p}}{E}\right)= \\
=\sum_{p=-T}^{T} p \cdot \chi^{e q}\left(\frac{V^{p}}{F}\right)+\sum_{p=-T}^{T}(e-p) \cdot \chi^{e q}\left(\frac{V^{p}}{F}\right)= \\
=e \cdot\left(\sum_{p=-T}^{T} \chi^{e q}\left(\frac{V^{p}}{F}\right)\right)=e \cdot \chi^{e q}\left(\frac{V}{F}\right)
\end{gathered}
$$

This ends the proof of Theorem 2.19

Definition 2.22. Let $U \subseteq M^{r}, V \subseteq M^{r}$. E, $F$ definable equivalence relations on $U$ and $V$, respectively. We define $E \times F$ on $U \times V$ by

$$
(u, v),\left(u^{\prime}, v^{\prime}\right) \in E \times F \Leftrightarrow u E u^{\prime} \wedge v F v^{\prime} .
$$

Note that $\frac{U}{E} \times \frac{V}{F}$ is in definable bijection with $\frac{U \times V}{E \times F}$, so we identify the two objects, and view $\frac{U}{E} \times \frac{V}{F}$ as a definable quotient.

Using this identification, Theorem 2.19 immediately implies:

Theorem 2.23. Assume $\frac{U}{E}, \frac{V}{F}$ are definable $M$-quotients with $V \subseteq M^{t}$ and $U \subseteq M^{r}$. Then

$$
\chi^{e q}\left(\frac{U}{E} \times \frac{V}{F}\right)=\chi^{e q}\left(\frac{U}{E}\right) \cdot \chi^{e q}\left(\frac{V}{F}\right)
$$

\section{Additional properties of $\chi^{e q}$}

Proposition 3.1. Assume $\frac{U}{E}$ is a definable quotient. If every E-class has Euler characteristic $k$, then $\chi(U)=k \cdot \chi^{e q}\left(\frac{U}{E}\right)$. In particular, $\chi(U)$ is a multiple of $k$.

Note that the "in particular" statement in the proposition concerns only the Euler characteristic of definable sets, but does not seem to immediately follow from the existing theory, without assuming that $\frac{U}{E}$ has a coding set.

Proof. There is definable bijection $f: \frac{U}{E} \rightarrow \frac{V}{F}$ such as every equivalence class $[v]_{F}$ is a cell, and

We will show

$$
\chi^{e q}\left(\frac{U}{E}\right)=\chi\left(V_{(1)}\right)-\chi\left(V_{(-1)}\right)
$$

$$
\chi(U)=\left(\chi\left(V_{(1)}\right)-\chi\left(V_{(-1)}\right)\right) \cdot k .
$$

Consider the following restrictions of $\mathrm{f}$ :

1. $f_{1}: \frac{U^{\prime}}{E} \rightarrow \frac{V_{(1)}}{F}$ when $U^{\prime}=\left\{u \in U \mid f\left([u]_{E}\right) \in \frac{V_{(1)}}{F}\right\}$ 
2. $f_{2}: \frac{U^{\prime \prime}}{E} \rightarrow \frac{V_{(-1)}}{F}$ when $U^{\prime \prime}=\left\{u \in U \mid f\left([u]_{E}\right) \in \frac{V_{(-1)}}{F}\right\}$.

Since $\frac{V}{F}=\frac{V_{(1)}}{F} \sqcup \frac{V_{(-1)}}{F}$, it follows that

By 2.10 we get

$$
\frac{U}{E}=\frac{U^{\prime}}{E} \sqcup \frac{U^{\prime \prime}}{E} .
$$

$$
\chi\left(U^{\prime}\right) \cdot 1=\chi\left(V_{(1)}\right) \cdot k \text { and } \chi\left(U^{\prime \prime}\right) \cdot(-1)=\chi\left(V_{(-1)}\right) \cdot k,
$$

SO

$\chi(U)=\chi\left(U^{\prime}\right)+\chi\left(U^{\prime \prime}\right)=\chi\left(V_{(1)}\right) \cdot k-\chi\left(V_{(-1)}\right) \cdot k=\left(\chi\left(V_{(1)}\right)-\chi\left(V_{(-1)}\right)\right) \cdot k$ as desired.

Finally, we note that our originally intended definition of $\chi\left(\frac{U}{E}\right)$, works well in the case where no class had Euler characteristic 0.

Proposition 3.2. Let $\frac{U}{E}$ be a definable quotient. If there is no equivalence class $[u]_{E}$ such that $\chi\left([u]_{E}\right)=0$, (i.e. $\left.U_{(0)}=\emptyset\right)$, then

$$
\chi^{e q}\left(\frac{U}{E}\right)=\sum \frac{\chi\left(U_{(k)}\right)}{k}
$$

Proof. Since $\frac{U}{E}=\sqcup_{k \neq 0} \frac{U_{(k)}}{E}$ it is enough to show for each $k \neq 0$ that $\chi^{e q}\left(\frac{U_{(k)}}{E}\right)=\frac{\chi\left(U_{(k)}\right)}{k}$. This is immediate from Proposition 3.1.

\section{REFERENCES}

[1] Lou van den Dries, Tame topology and o-minimal structures, London Mathematical Society Lecture Note Series, vol. 248, Cambridge University Press, Cambridge, 1998.

[2] Pantelis Eleftheriou, Ya'acov Peterzil, and Janak Ramakrishnan, Interpretable groups are definable, to appear: Journal of Mathematical Logic.

[3] William Johnson, A pathological o-minimal quotient, preprint (2014).

[4] Jerry Gagelman, Stability in geometric theories, Ann. Pure Appl. Logic 132 (2005), no. 2-3, 313-326.

[5] Ya'acov Peterzil, Constructing a group-interval in o-minimal structures, J. Pure Appl. Algebra 94 (1994), no. 1, 85-100.

[6] Anand Pillay, Some remarks on definable equivalence relations in O-minimal structures, J. Symbolic Logic 51 (1986), no. 3, 709-714.

[7] Adam W. Strzebonski, Euler characteristic in semialgebraic and other ominimal groups, J. Pure Appl. Algebra 96 (1994), no. 2, 173-201.

Department of Mathematics, University of Haifa, Haifa, Israel

E-mail address: simalda83@gmail.com

Department of Mathematics, University of Haifa, Haifa, Israel

E-mail address: kobi@math.haifa.ac.il 